\title{
Alimentación de los lenguados Cyclopsetta querna y Syacium latifrons en la costa de Jalisco y Colima, México
}

\author{
Mirella Saucedo Lozano ${ }^{1}$, Luz E. Rodríguez Ibarra ${ }^{2}$, Victor Landa Jaime ${ }^{1}$, Irma I. Guerrero Saucedo ${ }^{3}$ y \\ Jesús EMILIO Michel Morfin ${ }^{4}{ }^{*}$ \\ ${ }^{1}$ Departamento de Estudios para el Desarrollo Sustentable de Zonas Costeras, Centro Universitario de la Costa Sur, \\ Universidad de Guadalajara, Gómez Farías \#82, 48980 - San Patricio-Melaque, México. ${ }^{2}$ Centro de Investigación en Alimentos y Desarrollo, \\ A .C. Sábalo Cerritos s/n, 82010 A.P. 711 - Mazatlán, México. ${ }^{3}$ Sistema de Educación Media Superior, Escuela Preparatoria De Tonalá, \\ Universidad de Guadalajara, Calle Independencia No 232, 45400 - Colonia Centro Tonalá, México. ${ }^{4}$ Departamento de Ecología Aplicada, \\ Centro Universitario de Ciencias Biológicas y Agropecuarias, Universidad de Guadalajara, Camino Ing. Ramón Padilla Sánchez No 2100 , \\ 45510 - Zapopan, México. ORCID Mirella Saucedo Lozano (D) https://orcid.org/0000-0002-4566-6266, \\ Luz E. Rodríguez Ibarra (iD) https://orcid.org/0000-0001-8369-1000, Victor Landa Jaime (D) https://orcid.org/0000-0002-8464-1299, \\ Irma I. Guerrero Saucedo (D) https://orcid.org/0000-0003-3854-3994, Jesús Emilio Michel Morfin (D) https://orcid.org/0000-0002-9861-3360
}

\section{Marine and Fishery Sciences MAFIS}

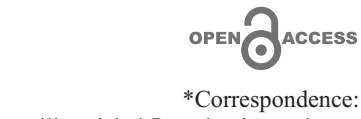

emilio.michel@academicos.udg.mx

Received: 21 June 2021 Accepted: 6 December 2021

ISSN 2683-7595 (print) ISSN 2683-7951 (online)

https://ojs.inidep.edu.ar

Journal of the Instituto Nacional de Investigación y Desarrollo Pesquero (INIDEP)

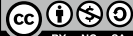

This work is licensed under a Creative Commons Attribution-

NonCommercial-ShareAlike 4.0 International License
RESUMEN. Los lenguados forman un componente importante como fauna de acompañamiento del camarón. El objetivo del presente trabajo consistió en analizar la alimentación de Cyclopseta querna y Syacium latifrons, con el fin de ampliar el conocimiento biológico de estas especies. Los ejemplares se colectaron en siete estaciones de muestreo con redes de arrastre de camarón. Se analizaron los estómagos de 196 individuos de C. querna y 313 de S. latifrons. El espectro trófico de C. querna estuvo constituido por 12 componentes alimentarios, mientras que para $S$. latifrons se registraron 24. Las presas principales de C. querna fueron los peces, mientras que para S. latifrons fueron los crustáceos, como por ejemplo, estomatópodos. Se observó una ligera variación ontogénica en la dieta de C. querna, no así en S. latifrons; sin embargo, en la dieta por sexos no se observaron diferencias. Los análisis de diversidad del espectro alimentario fueron menores de 0,5 para ambas especies. Se considera que en ambas especies la alimentación se da principalmente por la disponibilidad de recursos en las estaciones del año y por la capacidad de captura de presas. Por otra parte, la coexistencia de estas especies en una misma zona se da por diferencias en el consumo preferencial de sus presas al disminuir la competencia por alimento.

Palabras clave: Peces planos, trofodinámica, plataforma continental, Pacífico Este tropical.

Feeding of flatfish Cyclopsetta querna and Syacium latifrons on the coast of Jalisco and Colima, Mexico

ABSTRACT. Flatfish are important components of shrimp fisheries bycatch. The purpose of this study was to analyze feeding habits of Cyclopsetta querna and Syacium latifrons, with the aim of expanding the biological knowledge of these species. Specimens were collected in seven sampling stations with shrimp trawl nets. Stomachs of 196 individuals of C. querna and 313 of S. latifrons were analyzed. The trophic spectrum of C. querna consisted of 12 food components, while 24 were recorded for $S$. latifrons. The main prey for $C$. querna were fish, while for $S$. latifrons it was crustaceans, such as stomatopods. A slight ontogenetic trophic variation was observed in C. querna, not so in S. latifrons; however, no differences were observed considering only the diet by sex. Diversity analyses of the food spectrum were less than 0.5 for both species. It is considered that in both species feeding is given mainly by the seasonal availability of resources and by the ability to capture 
prey. On the other hand, the coexistence of these species in the same area is due to the difference in the preferential consumption of prey hence reducing the competition for food.

Key words: Flatfish, trophodynamic, continental shelf, Eastern tropical Pacific.

\section{INTRODUCCIÓN}

Dentro de las comunidades demersales, los peces constituyen un componente faunístico muy diversificado y de gran importancia económica y ecológica. Entre los grupos que forman un componente importante como fauna de acompañamiento del camarón se encuentran los lenguados o peces planos, pertenecientes al Orden Pleuronectiformes. Se ha reportado para el año 2018 un volumen en la producción pesquera mexicana de $5.331 \mathrm{t}$ de lenguados, de las cuales $5.215 \mathrm{t}$ correspondieron al Pacífico y $115 \mathrm{t}$ al Golfo de México y Caribe, lo que lo posiciona a este recurso pesquero en el lugar número 34 en la lista de las principales especies en volumen de producción pesquera nacional (Anuario estadístico de pesca y acuicultura 2018). Los lenguados son frecuentemente citados como organismos más representativos, comunes o frecuentes en este tipo de pesquerías (Rodríguez Ibarra 2006; Rodríguez Romero et al. 2008; López Martínez et al. 2010).

Los estudios para determinar los hábitos alimentarios cobran gran relevancia para comprender el papel biológico y ecológico que desempeña un organismo dentro del ecosistema, ya que el alimento constituye uno de los factores funcionales más importantes debido a que regula o afecta el crecimiento y reproducción, así como la forma en que se desarrolla su ciclo de vida, procesos que se dan a expensas de la energía que recibe del exterior (Nikolsky 1963; Lagler et al. 1984; Wootton 1990; Gerking 1994).

Existen pocos trabajos de alimentación en pleuronectiformes (Amezcua y Portillo 2010; Amezcua et al. 2010; Flores Ortega et al. 2013). La gran mayoría tratan otros aspectos biológicos y ecológicos como son la edad y crecimiento, distribución y abundancia, o solo se limitan a mencionarlos en listados taxonómicos (Murakami y Amoka 1992; Aceves-Medina et al. 2003; Khidir et al. 2005; Rodiles-Hernández et al. 2005; Rodríguez Ibarra 2006; Rojas y Zapata 2006; Rodríguez Romero et al. 2008; Tobón-López et al. 2008; López Martínez at al. 2010).

Cyclopsetta querna (Jordan y Bollaran, 1890) y Syacium latifrons (Jordan y Gilbert, 1882) no forman parte de una pesquería especial en la zona de estudio; sin embargo, aparecen frecuentemente en las capturas con redes de arrastre, y en ocasiones en grandes cantidades. Los estudios de alimentación y de población aportan datos para comprender la dinámica de las relaciones ecológicas que existen entre las especies, además de proporcionar las bases para establecer una correcta administración de los recursos pesqueros. No solo es importante generar este tipo de conocimiento para las especies de valor económico, sino también para todas aquellas con las que se relacionan ecológicamente, ya que una alteración en la dinámica de alguna especie podría afectar directa o indirectamente la supervivencia de otras especies. Dado lo anterior, en el presente estudio se fija el objetivo de proporcionar información sobre los hábitos alimentarios de C. querna y $S$. latifrons en la costa de Jalisco y Colima, México, mediante el análisis del contenido estomacal y la evaluación de la variabilidad trófica anual por grupos de talla y sexo.

\section{MATERIALES Y MÉTODOS}

Los ejemplares de C. querna y $S$. latifrons se recolectaron en la plataforma continental de Jalisco y Colima en un área comprendida desde la 
desembocadura del Río Cuitzmala, Punta Farallón, Estado de Jalisco $\left(19^{\circ} 21^{\prime} \mathrm{N}, 105^{\circ} 01^{\prime} \mathrm{W}\right)$ como límite norte, hasta Cuyutlán, Estado de Colima $\left(18^{\circ} 55^{\prime} \mathrm{N}, 105^{\circ} 01^{\prime} \mathrm{W}\right)$ al sur (Figura 1).

Las recolectas se realizaron a bordo del barco de investigación pesquera BIP-V del Departamento de Estudios para el Desarrollo Sustentable de Zonas Costeras de la Universidad de Guadalajara, en arrastres nocturnos realizados cuatrimestralmente en 1996, 1997 y 1998. Los arrastres se realizaron en siete sitios de muestreo definiendo cuatro niveles batimétricos a profundidades promedio de 20, 40, 60 y $80 \mathrm{~m}$, con un total de 28 arrastres por campaña. Adicionalmente durante el mismo período se llevaron a cabo muestreos mensuales en Bahía Navidad, Jalisco y Playa el Coco, Colima, a profundidades de 20 y $60 \mathrm{~m}$, siguiendo la misma metodología. En cada una de las profundi- dades se realizaron arrastres de aproximadamente $30 \mathrm{~min}$, utilizando redes de babor y estribor simultáneamente, a una velocidad media de dos nudos.

Para cada ejemplar se registró la longitud total (LT, cm). Los estómagos se conservaron en solución de formalina al $10 \%$ hasta su posterior análisis. Los diversos componentes de la dieta fueron identificados hasta el nivel taxonómico más bajo posible mediante el uso de claves y de literatura especializada. Para invertebrados, Rathbun (1930), Brusca (1980), Hendrickx y SalgadoBarragán (1991), Hendrickx (1995a, 1995b), Pérez Farfante y Kensley (1997). Para peces, Allen y Robertson (1994), Fischer et al. (1995), Nelson (2006). La vigencia de todos los nombres científicos fue verificada de acuerdo con la pagina WORMS considerando los nuevos cambios taxonómicos propuestos en años recientes.

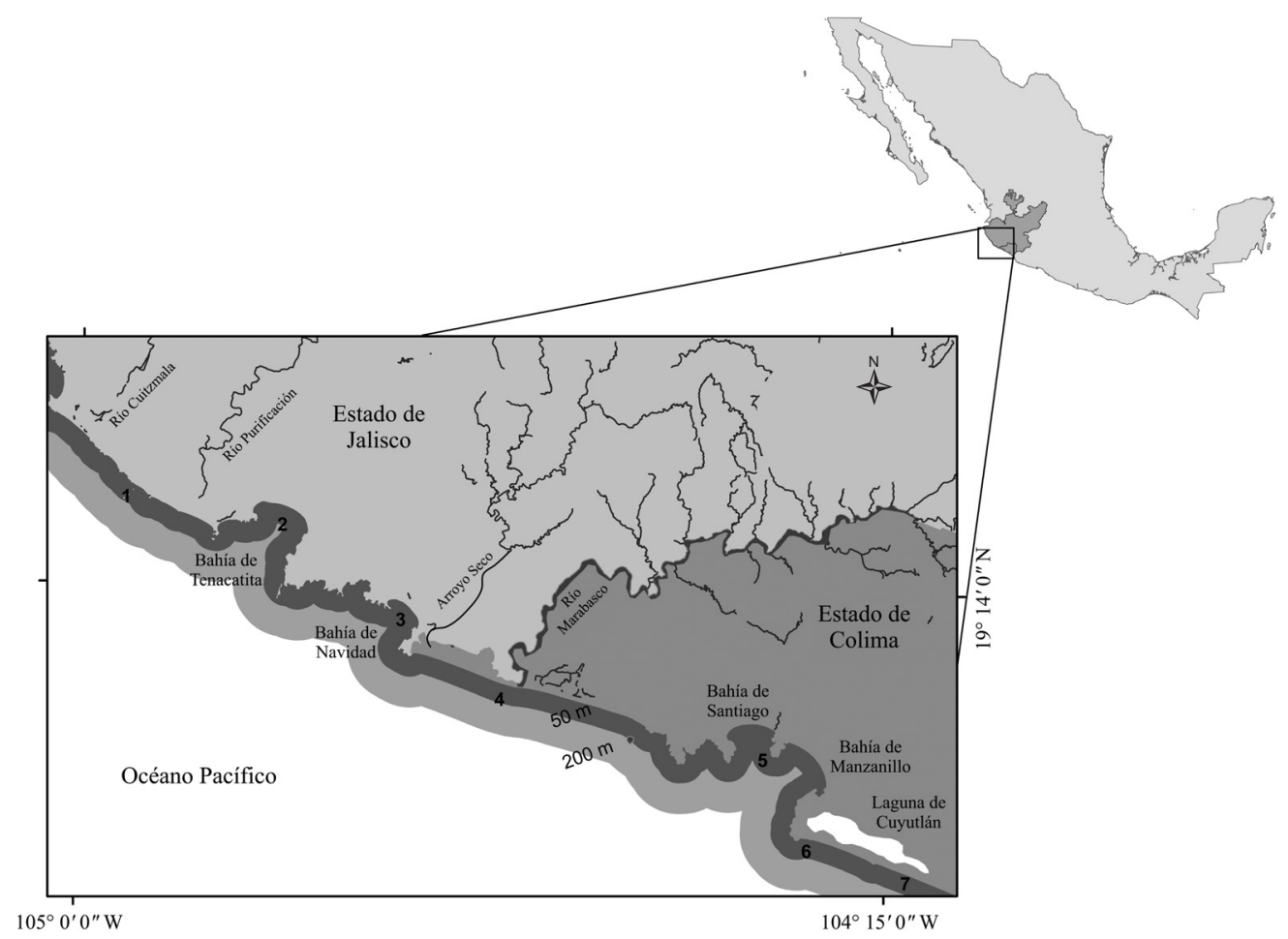

Figura 1. Área de estudio indicando los sitios de muestreo: (1) Cuitzmala, (2) Tenacatita, (3) Bahía de Navidad, (4) El Coco, (5) Manzanillo-Santiago, (6) Tepalcates, (7) Cuyutlán. Costa de Jalisco y Colima, México.

Figure 1. Study area with sampling sites: (1) Cuitzmala, (2) Tenacatita, (3) Bahía de Navidad, (4) El Coco, (5) ManzanilloSantiago, (6) Tepalcates, (7) Cuyutlán. Jalisco and Colima coast, Mexico. 
Las entidades alimentarias del contenido estomacal fueron contadas y pesadas. Para describir la dieta de las especies se utilizó el método numérico $(\% \mathrm{~N})$, el método gravimétrico $(\% \mathrm{~W})$ (Rosecchi y Novaze 1987) y el método de frecuencia de ocurrencia (\%FO) (Pinkas et al. 1971). La importancia relativa de cada especie presa de los lenguados fue establecida por el Índice de Importancia Relativa Específico para Presas $(\% \mathrm{PSIRI})=\% \mathrm{~F}_{i} *\left(\% \mathrm{PN}_{i}+\right.$ $\left.\% \mathrm{PW}_{i}\right) / 2$ (Brown et al. 2012), donde $\% \mathrm{~F}_{i}$ es el porcentaje del número de estómagos donde se encontró la presa $i, \% \mathrm{PN}_{i}$ es el porcentaje en número específico para la presa $i$ y $\% \mathrm{PW}_{i}$ es el porcentaje en peso específico para la presa $i$.

Para evaluar si el tamaño de la muestra (número de estómagos) es suficiente para describir la dieta de los lenguados, se realizó una curva de acumulación de presas para cada especie a partir del número total de especies presa identificadas mediante el uso del software EstimateS 9.1.0 (Colwell 2019), basado en 100 aleatorizaciones sin reemplazo para asegurar que las curvas alcanzaran un valor asintótico. El valor del índice de Shannon (Magurran 2004) se representó en función del número acumulado de estómagos examinados (Figueiredo et al. 2005). Cada curva de diversidad trófica se consideró asintótica si al menos dos valores anteriores a la diversidad trófica (H'TOT) estuvieran en un intervalo H'TOT \pm 0,05 H'TOT (Alonso et al. 2002).

Con los valores de la longitud total de C. querna y $S$. latifrons, se determinó el número de intervalos de talla. Para los intervalos de clase se utilizó la fórmula de Sturges (1926): $\mathrm{k}=1+3,322$ $\left(\log _{10} \mathrm{n}\right)$, donde, $\mathrm{k}=$ número de intervalos de clase y $\mathrm{n}=$ número total de valores. Posteriormente, se procedió a determinar la amplitud del intervalo, el cual se calculó con la fórmula propuesta por Daniel (1997): $\mathrm{W}=\mathrm{R} / \mathrm{k}$, donde $\mathrm{W}=$ amplitud del intervalo y $\mathrm{R}=$ valor máximo menos el valor mínimo. Se denominaron cuatro grupos de talla para $C$. querna $(0-15 \mathrm{~cm}, 15,1-30 \mathrm{~cm}$, $30,1-45 \mathrm{~cm}$ y $45,1 \mathrm{~cm}<$ ) y dos grupos para $S$. latifrons $(0-15 \mathrm{~cm}$ y 15,1-30 cm). Esta aproximación al número y amplitud de intervalos de talla ha sido aplicada anteriormente en otras especies de peces (Bocanegra-Castillo et al. 2000; Kouamelan et al. 2000; Raymundo Huizar y Saucedo Lozano 2008), considerándose apropiado para explicar variaciones en la dieta respecto a la talla.

Se realizó una evaluación de la amplitud del espectro trófico (amplitud de nicho), con el fin de determinar el grado de especialización en la dieta. Para tal efecto, se utilizó la medida de Levins (1968), estandarizada por el método de Hespenheiden (1975), utilizando una escala de 0 a 1 , considerándose como especialista cuando el valor es cercano a cero y generalista cuando el valor es cercano a uno (Krebs 1989):

$$
B=\frac{1}{\sum_{i=1}^{n} P_{j}^{2}} \quad B_{a}=\frac{B-1}{n-1}
$$

donde $B$ es la medida de Levins sobre la amplitud de la dieta; $B_{a}$ la medida de Levins estandarizada; $P_{j}$ equivale a la proporción con la cual cada categoría de presa contribuye a la dieta y $n$ es el número total de recursos alimenticios.

Se estableció el grupo de presas de mayor importancia en la dieta, así como aquellos que son consumidos de manera accidental, con el Índice Alimentario (IA) modificado por Lauzanne (1975), $\mathrm{IA}=\% \mathrm{~F} \times \% \mathrm{P}(\% \mathrm{~A}) / 100$. Al relacionar las preferencias alimentarias $(\% \mathrm{FO})$ y la importancia relativa de las presas en peso $(\% \mathrm{P})$ o área $(\% \mathrm{~A})$, se integran los artículos alimentarios, lo que permite distinguir al alimento preferencial de aquel cuyo consumo es de baja frecuencia (Rosechi y Novaze 1987). Los valores de IA varían de 0 a $100 \%$ y se categorizar en función de su importancia relativa como: 1) alimento preferencial (IA $>50), 2$ ) secundario $(25<\mathrm{IA}>50), 3)$ frecuente $(10<\mathrm{IA}>$ 25) y 4) accidental (IA < 10) (Lauzanne 1975).

Por último, se realizó una evaluación cuantitativa del grado de uso de los recursos alimentarios que comparten ambas especies al coexistir en un 
mismo hábitat, a través de un análisis de conglomerados por medio del índice de Morisita (1959), modificado por Horn (1966):

$\operatorname{Im}=\left(2 \sum_{i=1}^{n} X i Y i\right) /\left(\sum_{i=1}^{n} X i^{2}+\sum_{i=1}^{s} Y i^{2}\right.$

donde Im es el índice de traslape entre dos especies depredadoras, $n$ el número total de categorías alimentarias, $X i$ e $Y i$, son las proporciones de la dieta total de las especies $X$ e $Y$, tomadas de la categoría alimentaria i con respecto al total de los recursos utilizados por ambas especies $(i=1,2$, $3, \ldots$ n). El índice varía de 0 , cuando no hay elementos de la dieta en común, a 1 cuando todos los artículos alimentarios son comunes. Para corroborar los resultados de traslape de la dieta o las posibles diferencias de alimentación entre ambas especies, se utilizó la prueba de $X^{2}$ (Ludwing y Reynolds 1988).

\section{RESULTADOS}

Durante las campañas realizadas, se recolectaron un total de 509 ejemplares. De éstos, 196 correspondieron a C. querna, de los cuales $115(58,67 \%)$ presentaron contenido estomacal y $81(41,33 \%)$ estómagos vacíos. S. latifrons totalizó 313 organismos, de los cuales $238(76,04 \%)$ presentaron contenido estomacal y $75(23,96 \%)$ se encontraron con el estómago vacío. El rango de longitud total varío entre 2,8 y $48,8 \mathrm{~cm}$ para C. querna, y entre 6,5 y 26 cm para S. latifrons (Tabla 1).

Las curvas de acumulación de las presas registradas para C. querna y $S$. latifrons (Figura 2 A y B) muestran una tendencia a la asíntota, por lo que se considera que el tamaño de muestra analizado es adecuado. Por ende, las inferencias obtenidas de este estudio son representativas de la población de lenguados.

Tabla 1. Número de ejemplares de Ciclopsetta querna y Syacium latifrons con presencia de contenido estomacal, en función de su talla y sexo.

Table 1. Number of specimens of Ciclopsetta querna and Syacium latifrons with presence of stomach content by size and sex classes.

\begin{tabular}{|c|c|c|c|c|c|c|}
\hline & \multicolumn{3}{|c|}{ Cyclopseta querna } & \multicolumn{3}{|c|}{ Syacium latifrons } \\
\hline & 1996 & 1997 & 1998 & 1996 & 1997 & 1998 \\
\hline \multicolumn{7}{|l|}{ Grupos de tallas } \\
\hline $0-15$ & 5 & & & & & \\
\hline $15,1-30$ & 25 & 3 & 0 & 24 & 22 & 46 \\
\hline $30,1-45$ & 20 & 17 & 13 & 67 & 15 & 64 \\
\hline $45>$ & 0 & 9 & 19 & 0 & 0 & 0 \\
\hline Total número de organismos & 50 & 4 & 0 & 0 & 0 & 0 \\
\hline \multicolumn{7}{|l|}{ Sexos } \\
\hline Indeterminados & 7 & 0 & 0 & 0 & 0 & 0 \\
\hline Hembras & 17 & 11 & 17 & 49 & 18 & 41 \\
\hline Machos & 26 & 22 & 15 & 46 & 19 & 69 \\
\hline
\end{tabular}



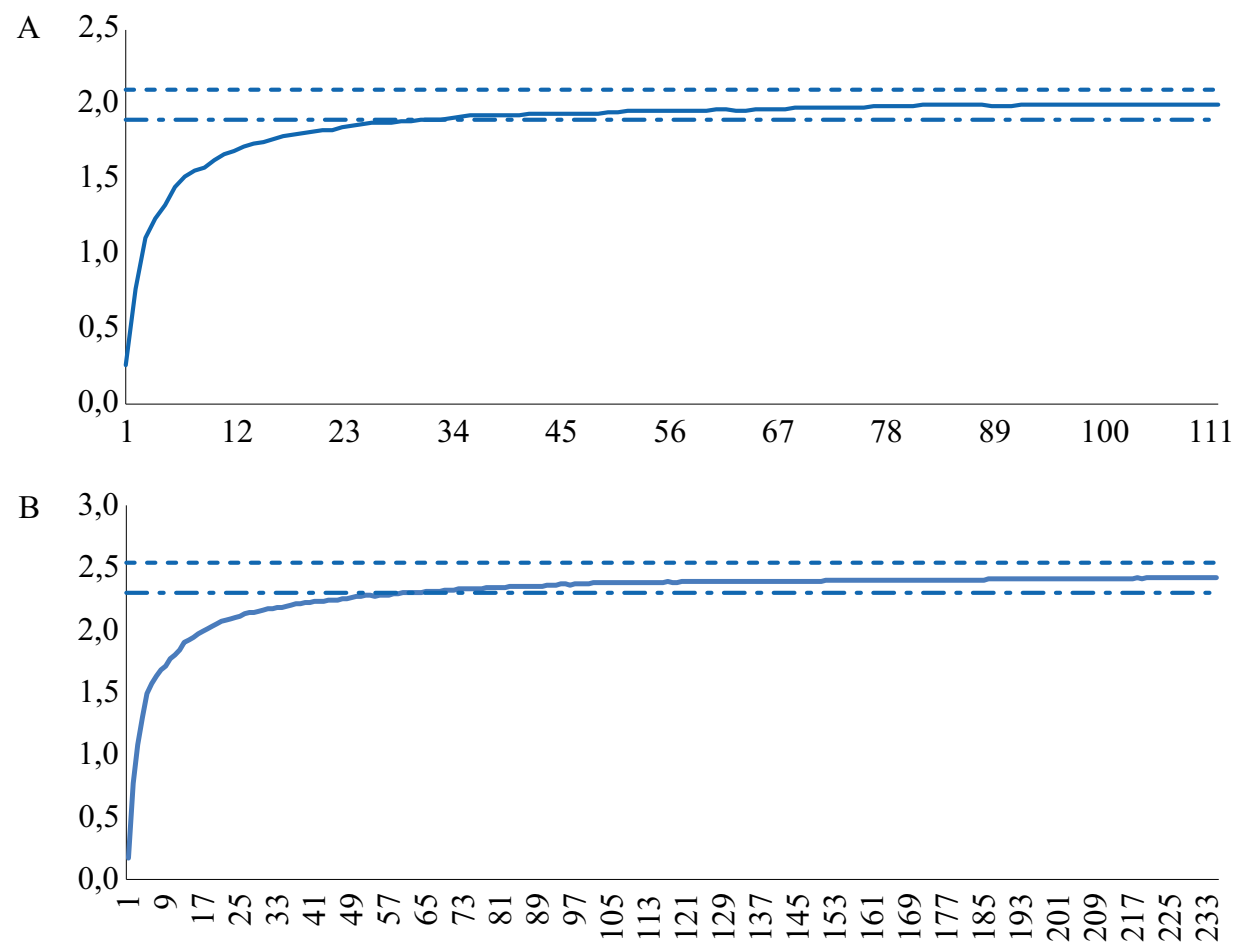

_ Shannon media _- Mínimos _. - Máximos

Figura 2. Curva de acumulación de diversidad trófica. Las líneas intermitentes indican el $\mathrm{H}_{\max }^{\prime} \pm 0,05 \mathrm{H}_{\max }$. A) Para Cyclopsetta querna. B) Para Syacium latifrons.

Figure 2. Trophic diversity accumulation curve. Flashing lines indicate $H^{\prime}{ }_{\max } \pm 0.05 H^{\prime}{ }_{\max }$. A) For Cyclopsetta querna. B) For Syacium latifrons

El espectro trófico de $C$. querna estuvo constituido por 12 componentes alimentarios pertenecientes a dos taxas (peces y crustáceos), mientras que para $S$. latifrons se registraron 24 componentes alimentarios pertenecientes a tres taxas (peces, crustáceos y moluscos) (Tabla 2).

Para fines de la descripción general de la dieta, los componentes alimentarios se agruparon en cinco categorías para C. querna (peces, estomatópodos, portúnidos, peneidos y otros crustáceos) y seis para $S$. latifrons (peces, estomatópodos, portúnidos, peneidos, otros crustáceos y otros molus$\cos )$. El grupo de peces fue el dominante para $C$. querna en los tres años en número $(73,53 \%$, $81,82 \%$ y $75,00 \%)$, en peso $(92,07 \%, 94,30 \%$, 93,59\%), en índice de importancia relativa específico para presas $(82,80 \%, 88,01 \%, 84,29 \%)$
(Figura 3). Sin embargo, en la frecuencia de ocurrencia, los mayores porcentajes los presentaron los restos de crustáceos $(38,00 \%)$ en 1996, la Familia Congridae (36,36\%) en 1997 y los restos de peces $(52,50 \%)$ en 1998 (Tabla 2).

En cuanto a $S$. latifrons, solo en 1996 se registraron variaciones, ya que los estomatópodos presentaron el mayor porcentaje en peso $(44,29 \%)$ mientras que los peneidos lo presentaron en número $(71,61 \%)$ y en el índice de importancia relativa específico para presas (45,99\%). En 1997 y 1998, dominaron los estomatópodos con porcentajes de $68,48 \% \mathrm{~W}, 61,11 \% \mathrm{~N}, 64,80 \%$ PSIRI y $63,05 \% \mathrm{~W}$, $62,79 \% \mathrm{~N}, 62,92 \% \mathrm{PSIRI}$, respectivamente (Figura 4). En lo que respecta a la frecuencia de ocurrencia, los restos de estomatópodos presentaron el mayor porcentaje en 1996 y 1998 con 20,88\% y 


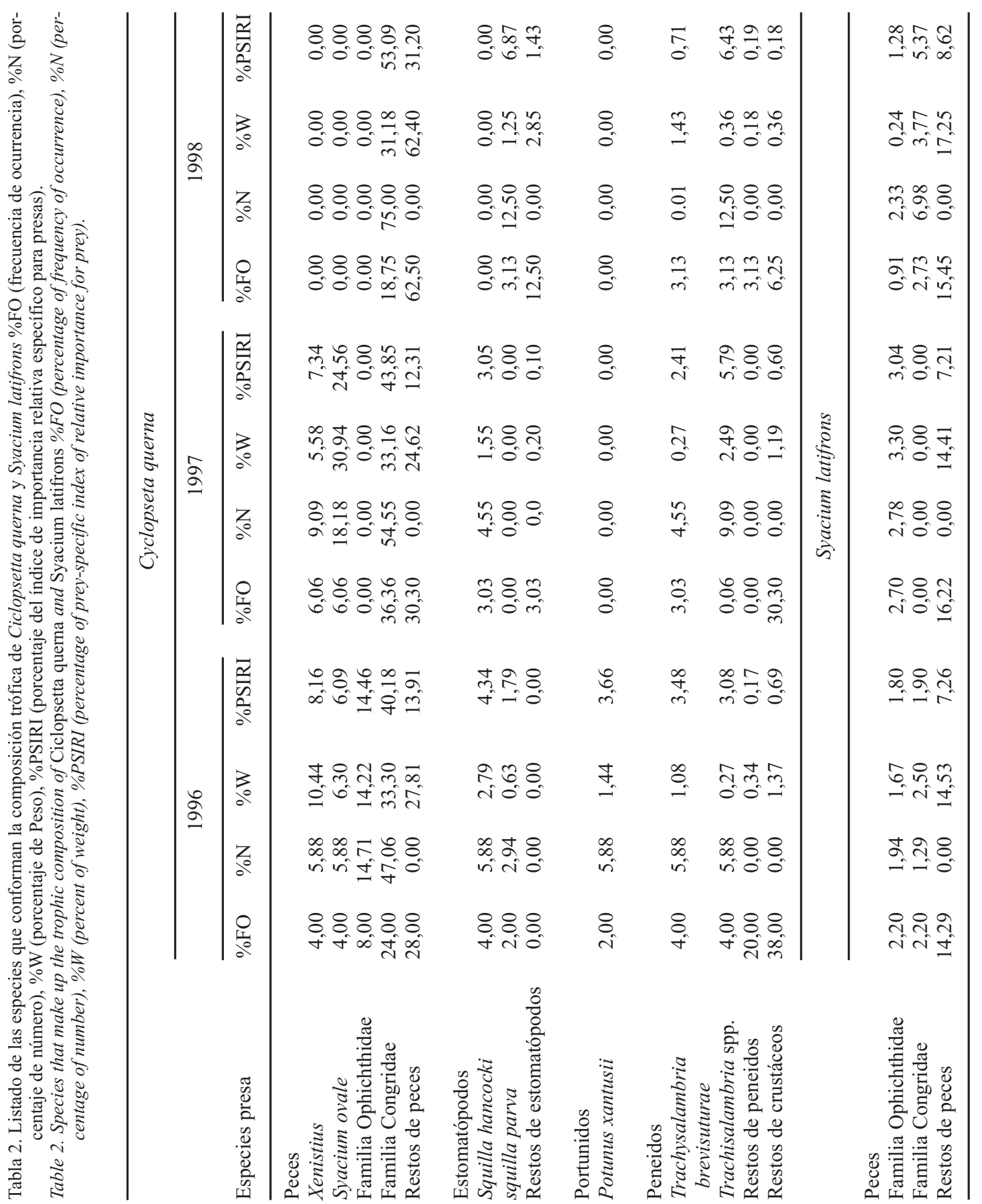




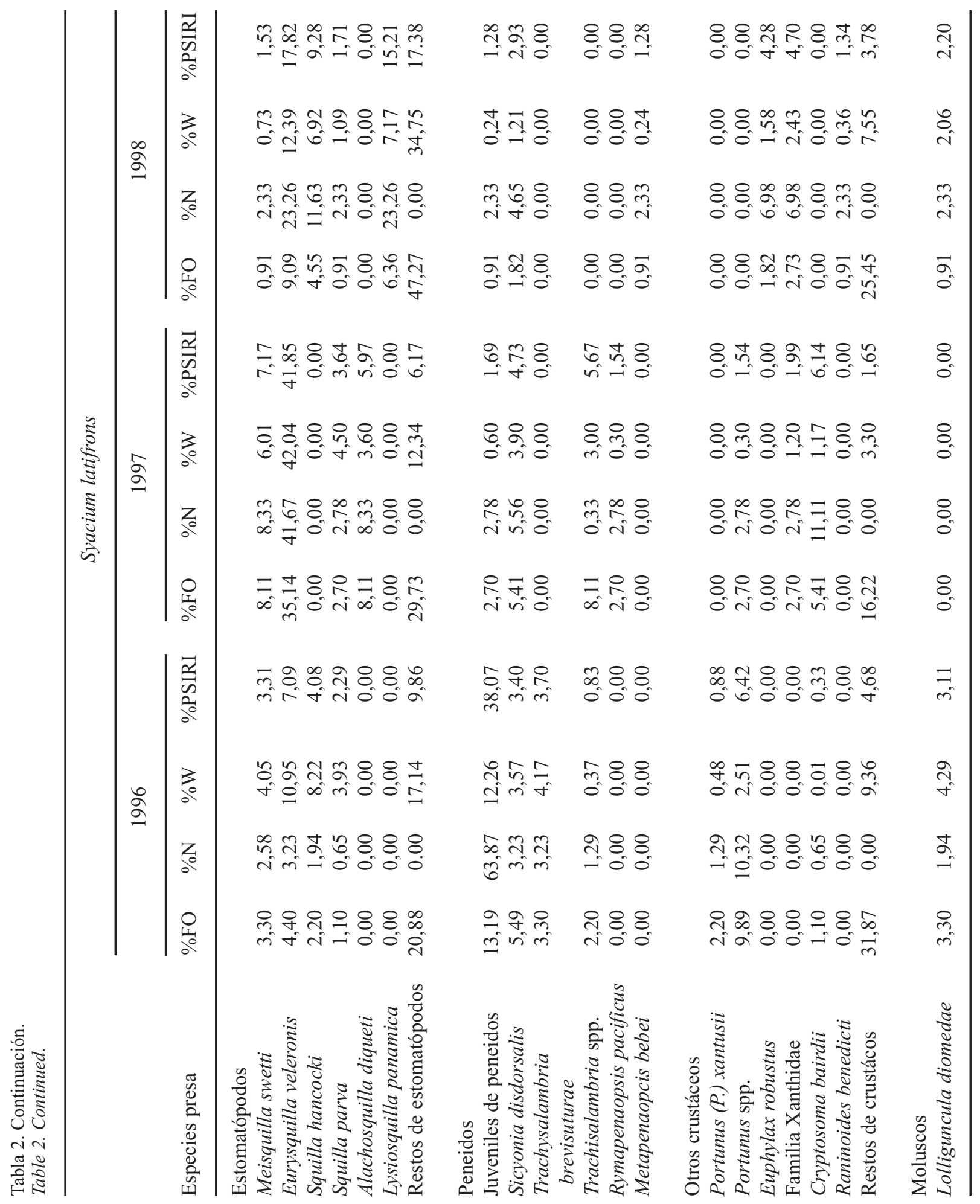




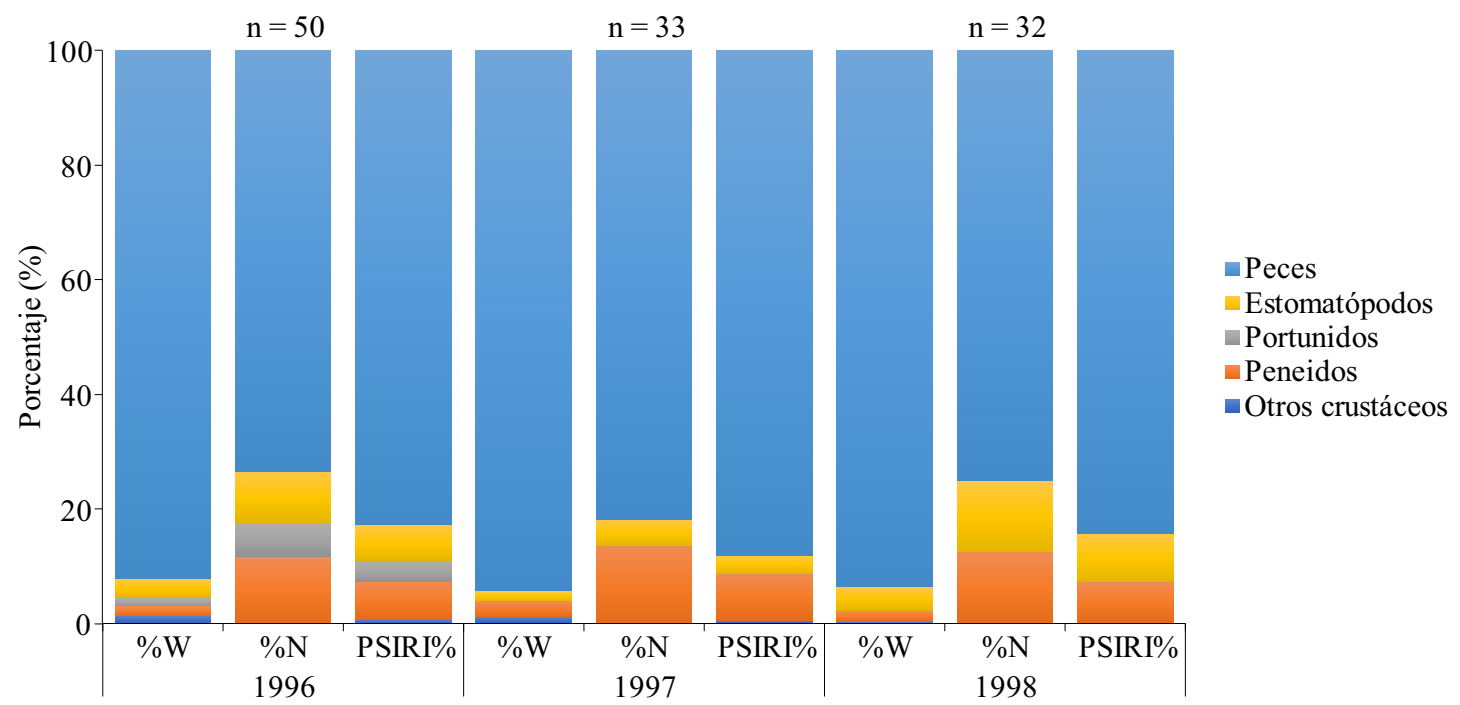

Años, métodos e índice

Figura 3. Variación del espectro alimentario de acuerdo con el porcentaje en peso $(\% \mathrm{~W})$, porcentaje en número $(\% \mathrm{~N})$ y porcentaje del índice de importancia presa específica (\%PSIRI), para la dieta de Cyclopsetta querna durante los tres años del estudio.

Figure 3. Variation of the alimentary spectrum according to the percentage in weight (\%W), percentage in number (\%N) and percentage of prey-specific index of relative importance (\%PSIRI), for food items of Cyclopsetta querna during three years of study.

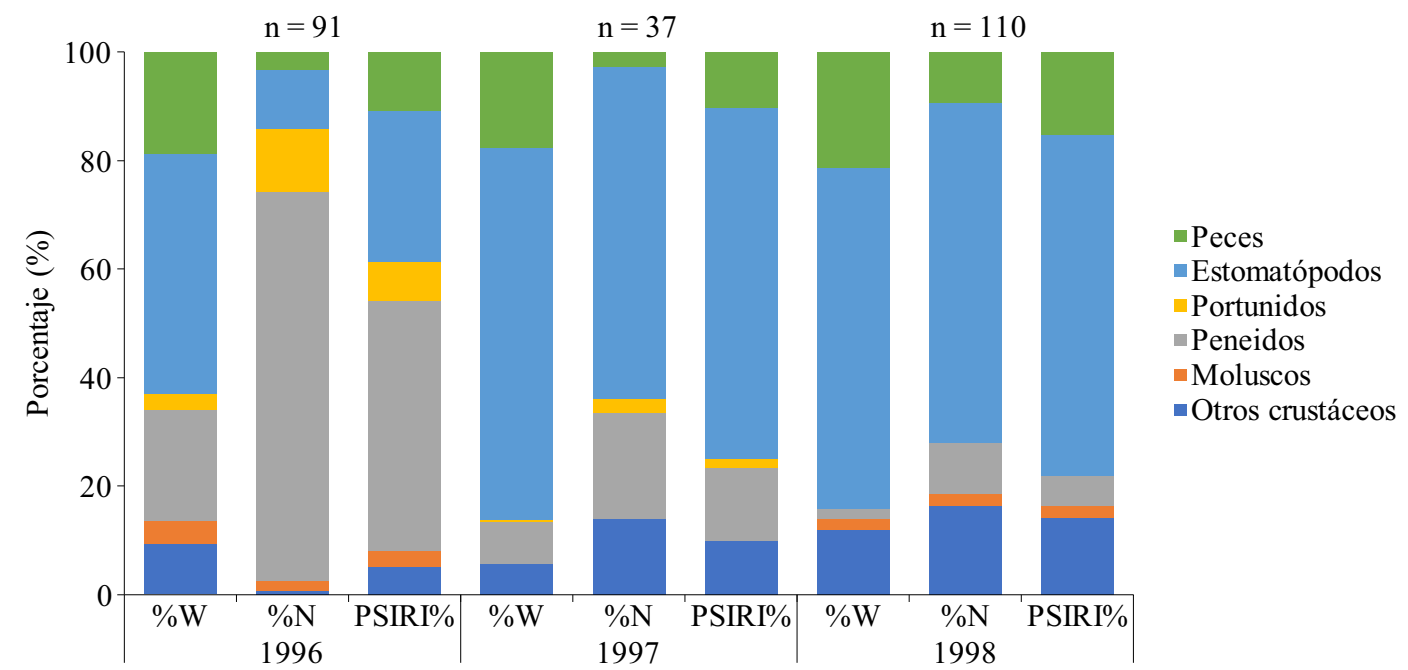

Años, métodos e índice

Figura 4. Variación del espectro alimentario de acuerdo con el porcentaje en peso $(\% \mathrm{~W})$, porcentaje en número $(\% \mathrm{~N})$ y porcentaje del índice de importancia presa específica (\%PSIRI), para la dieta de Syacium latifrons durante los tres años del estudio.

Figure 4. Variation of the alimentary spectrum according to the percentage in weight (\%W), percentage in number (\%N) and percentage of prey-specific index of relative importance (\%PSIRI), for food items of Syacium latifrons during three years of study. 
47,27\%, respectivamente, y para 1997 fue el grupo de otros crustáceos con 45,06\%, seguido de Eurysquilla veleronis con $35,14 \%$ (Tabla 2).

\section{Variación anual en la dieta}

C. querna consumió en los tres años preferentemente peces de la Familia Congridae con valores de 40,18, 43,85 y 53,09\%PSIRI, respetivamente. El segundo componente en importancia en 1996 fueron los peces de la Familia Ophichthidae (14,46 \%PSIRI), en 1997 fue S. ovale (24,56 $\%$ PSIRI), y por último en 1998 los restos de peces $(31,20 \%$ PSIRI) (Figura 5).

El lenguado $S$. latifrons presentó en 1996 como presa principal a los juveniles de peneidos (38.07 $\%$ PSIRI), seguido de restos de estomatópodos (9,86 \%PSIRI). Para 1997 fue E. veleronis con $41,85 \%$ PSIRI, seguida de restos de peces $(7,21$ \%PSIRI), y en 1998 nuevamente aparece $E$. veleronis como la presa más importante $(17,82$ \%PSIRI), seguida por restos de estomatópodos $(17,38 \%$ PSIRI) (Figura 6).

\section{Variación ontogénica en la dieta}

Se observaron variaciones en la dieta de $C$. querna relacionadas con la talla (Figura 7). Así, para 1996 en el primer grupo de talla $(0-15 \mathrm{~cm}$ LT) dominaron los restos de crustáceos $(90,15$ \%PSIRI); en el segundo grupo (15,1-30 cm LT) las presas más importantes fueron los peces de la Familia Congridae (51,83\%PSIRI) y restos de peces $(13,28 \%$ PSIRI); para el tercer grupo $(30,1-45 \mathrm{~cm} \mathrm{LT})$ nuevamente fueron peces de la Familia Congridae (24,85\%PSIRI) y Xenistius californiensis (19,24\%PSIRI); y para el cuarto grupo $(45,1<\mathrm{cm} \mathrm{LT})$ no se registraron organismos. En 1997, las presas más importantes para los organismos de tallas de 0 a $15 \mathrm{~cm}$ LT fueron $X$. californiensis $(87,71 \%$ PSIRI) y restos de crustáceos (12,29\%PSIRI); en los de talla 15,1$30 \mathrm{~cm}$ LT los peces de la Familia Congridae (56,68 \%PSIRI) y $S$. ovale $(17,27 \%$ PSIRI); para el tercer grupo $(30,1-45 \mathrm{~cm}$ LT) las especies dominantes fueron nuevamente $S$. ovale $(37,77$ $\%$ PSIRI) y peces de la Familia Congridae $(32,70$

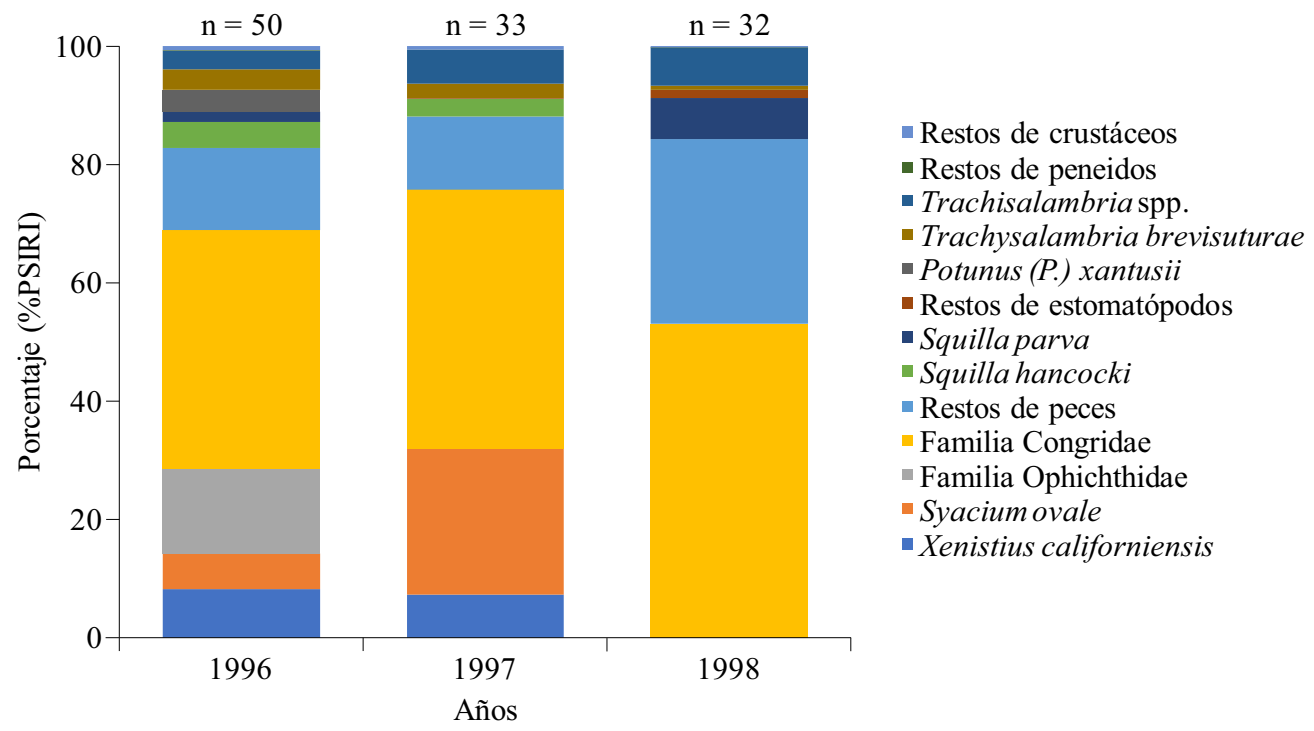

Figura 5. Porcentaje del índice de importancia presa específica (\%PSIRI) de las especies presas para la dieta de Cyclopsetta querna durante los tres años de estudio.

Figure 5. Percentage of prey-specific index of relative importance (\%PSIRI) for food items of Cyclopsetta querna during three years of study. 


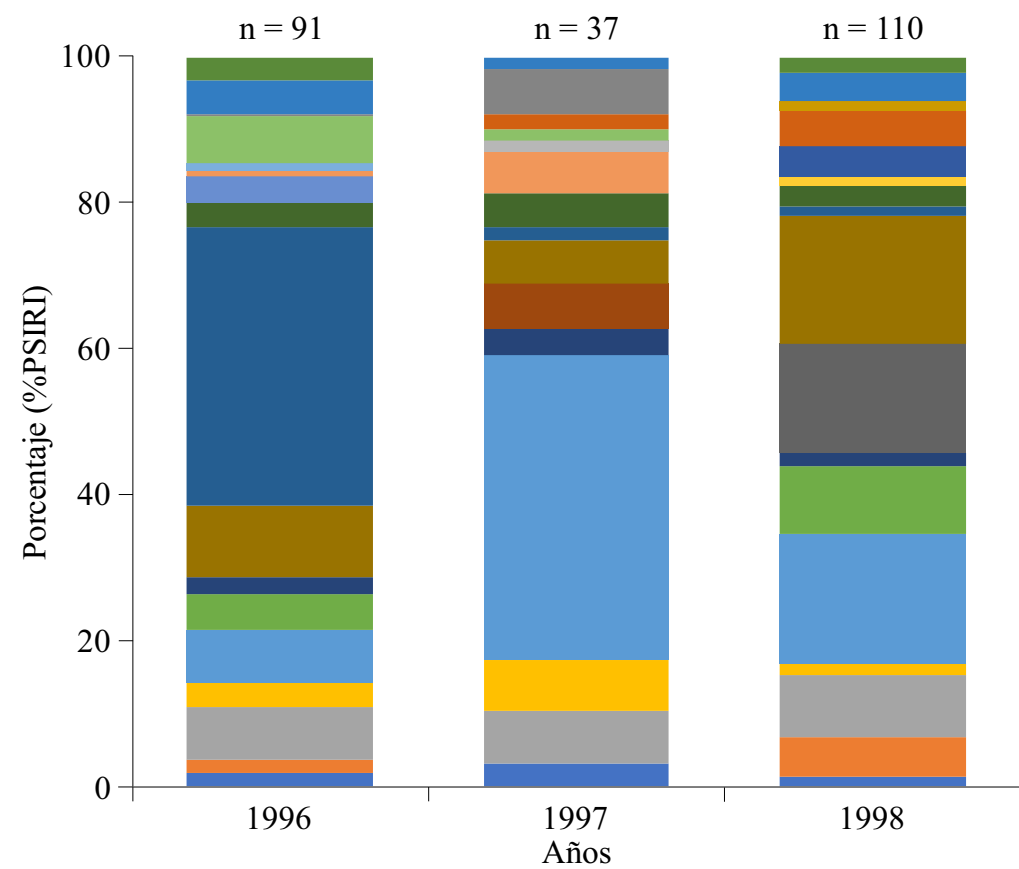

- Lolliguncula diomedae

- Restos de crustáceos

- Raninoides benedicti

- Cryptosoma bairdii

- Familia Xanthidae

- Euphylax robustus

- Portunus spp.

- Portunus (P.) xantusii

- Metapenaopcis bebei

- Rymapenaopsis pacificus

- Trachisalambria spp.

- Trachysalambria brevisuturae

- Sicyonia disdorsalis

- Juveniles de peneidos

- Restos de estomatópodos

- Lysiosquilla panamica

- Alachosquilla diqueti

- Squilla parva

- Squilla hancocki

- Eurysquilla veleronis

- Meisquilla swetti

- Restos de peces

- Familia Congridae

- Familia Ophichthidae

Figura 6. Porcentaje del índice de importancia presa específica (\%PSIRI) de las especies de presas para la dieta de Syacium latifrons durante los tres años de estudio.

Figure 6. Percentage of prey-specific index of relative importance (\%PSIRI) for food items of Syacium latifrons during three years of study.

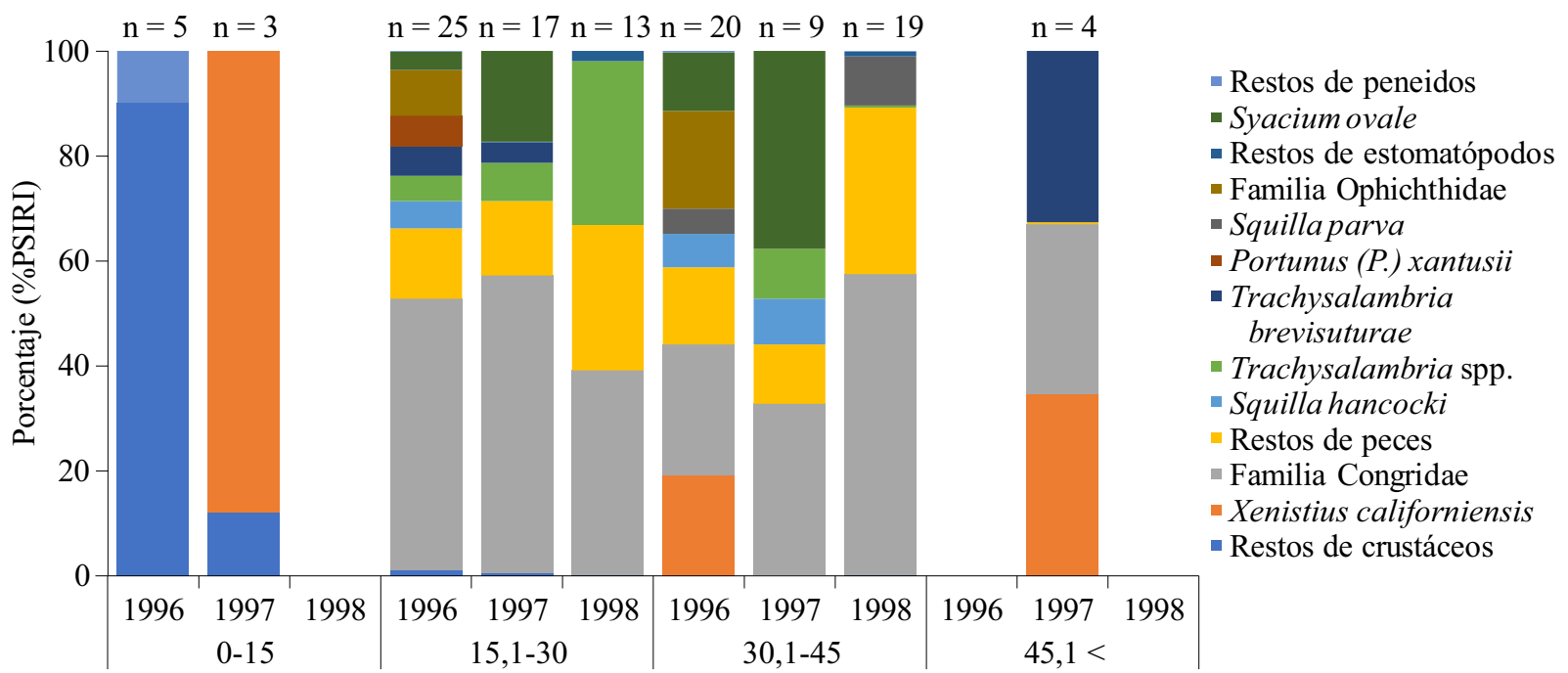

Años y grupos de talla $(\mathrm{cm})$

Figure 7. Porcentaje del índice de importancia presa específica (\%PSIRI), con respecto a los grupos de talla (cm LT) de Cyclopsetta querna durante los tres años de estudio.

Figure 7. Percentage of prey-specific index of relative importance (\%PSIRI) for food items of Cyclopsetta querna by length class (cm TL) during three years of study. 
$\%$ PSIRI). Para el último grupo $(45,1<)$ dominaron como presas $X$. californiensis $(34,72$ \%PSIRI) y Trachysalambria brevisuturae (32,64 \%PSIRI). En 1998 no se registraron organismos en los grupos de 0 a $15 \mathrm{~cm}$ LT y en el de mayores de 45,11 cm LT. No obstante, para los organismos del grupo de tallas 15,1-30 cm LT y de 30,1-45 cm LT, las presas más importantes fueron los peces de la Familia Congridae (39,00\% y 57,35 \%PSIRI) y Trachysalambria spp. (31,20\%PSIRI), y restos de peces $(31,71$ $\%$ PSIRI), respectivamente.

Con respecto a la variación ontogénica para $S$. latifrons, solo se registraron dos grupos de tallas en los tres años (0-15 cm y 15,1-30 cm). En 1996 dominaron los restos de crustáceos $(22,58$ $\%$ PSIRI), seguido de Meiosquilla swetti (19,85 \%PSIRI). En el segundo grupo, las presas más importantes fueron los juveniles de peneidos $(39,28 \%$ SPIRI $)$ y restos de estomatópodos $(9,84$ $\%$ PSIRI). Para 1997, la dieta de organismos pequeños (0-15 cm LT) estuvo dominada por $E$. veleronis (54,38 \%PSIRI) y M. swetti $(10,51$ $\%$ PSIRI), mientras que en el segundo grupo (15,1-30 cm LT) primaron los restos de peces (18,44 \%PSIRI), seguidos de E. veleronis (16,56 $\%$ PSIRI). Finalmente, para 1998, en el grupo de 0-15 cm LT la presa principal fue Lysiosquilla panamica $(19,95 \%$ PSIRI) y restos de estomatópodos (19,61 \%PSIRI), mientras que para el grupo de 15,1-30 cm LT fue E. veleronis $(17,09$ $\%$ PSIRI), seguida de restos de estomatópodos (16,60 \%PSIRI) (Figura 8).

\section{Variación por sexos}

La dieta de $C$. querna no muestra diferencias significativas entre sexos, ya que en los tres años, tanto para organismos inmaduros como para hembras y machos, las presas más importantes fueron los peces entre los que dominaron los de las familias Congridae y Ophichthydae, X. californiensis, $S$. ovale y restos de otros peces (Figura $9)$. Por su parte, $S$. latifrons presenta un compor- tamiento similar debido a que los grupos de presas dominantes fueron los crustáceos, con algunas variaciones en cuanto a las especies consumidas. Para 1996, las presas principales fueron los juveniles de peneidos, tanto en hembras $(36,89$ \%PSIRI) como en machos (39,77 \%PSIRI). En 1997 , la presa principal fue E. veleronis en hembras $(36,22 \%$ PSIRI) y machos $(49,72 \%$ PSIRI). Por último, en 1998 las hembras consumieron principalmente a $E$. veleronis $(18,98 \% \mathrm{PSIRI})$, y en los machos el mayor porcentaje lo representaron los restos de estomatópodos (18,88\%PSIRI) (Figura 10).

\section{Amplitud de nicho}

La diversidad del espectro alimentario, considerada también como la amplitud del nicho trófico, indica el grado de especialización de los hábitos alimentarios de una especie. Valores cercanos a 0 indican que la especie es especialista en su dieta. En los tres años, la máxima diversidad observada fue menor de 0,5 , por lo que se puede apreciar visualmente que las dos especies presentan cierto grado de especialización. En 1996, S. latifrons mostró la mayor amplitud en cuanto al valor del índice calculado en porcentaje en peso $(0,45)$ (Figura $11 \mathrm{~A})$. En lo que respecta al índice porcentaje en número, C. querna presentó para 1996 y 1997 valores $\leq 0,26$, mientras que para $S$. latifrons los valores más altos fueron en 1998 con 0,25 (Figura $11 \mathrm{~B}$ ).

\section{Índice alimentario}

Los grupos que constituyen el espectro trófico de C. querna para 1996 y 1997 son principalmente peces de la Familia Congridae y la categoría de restos de peces, considerados como secundarios (Tabla 3). En conjunto, alcanzan entre el 80 y $90 \%$ del valor del índice. Para 1998, los restos de peces dominaron como alimento preferencial $(86,13 \%)$, seguido de la Familia Congridae $(12,91 \%)$ como alimento frecuente. 


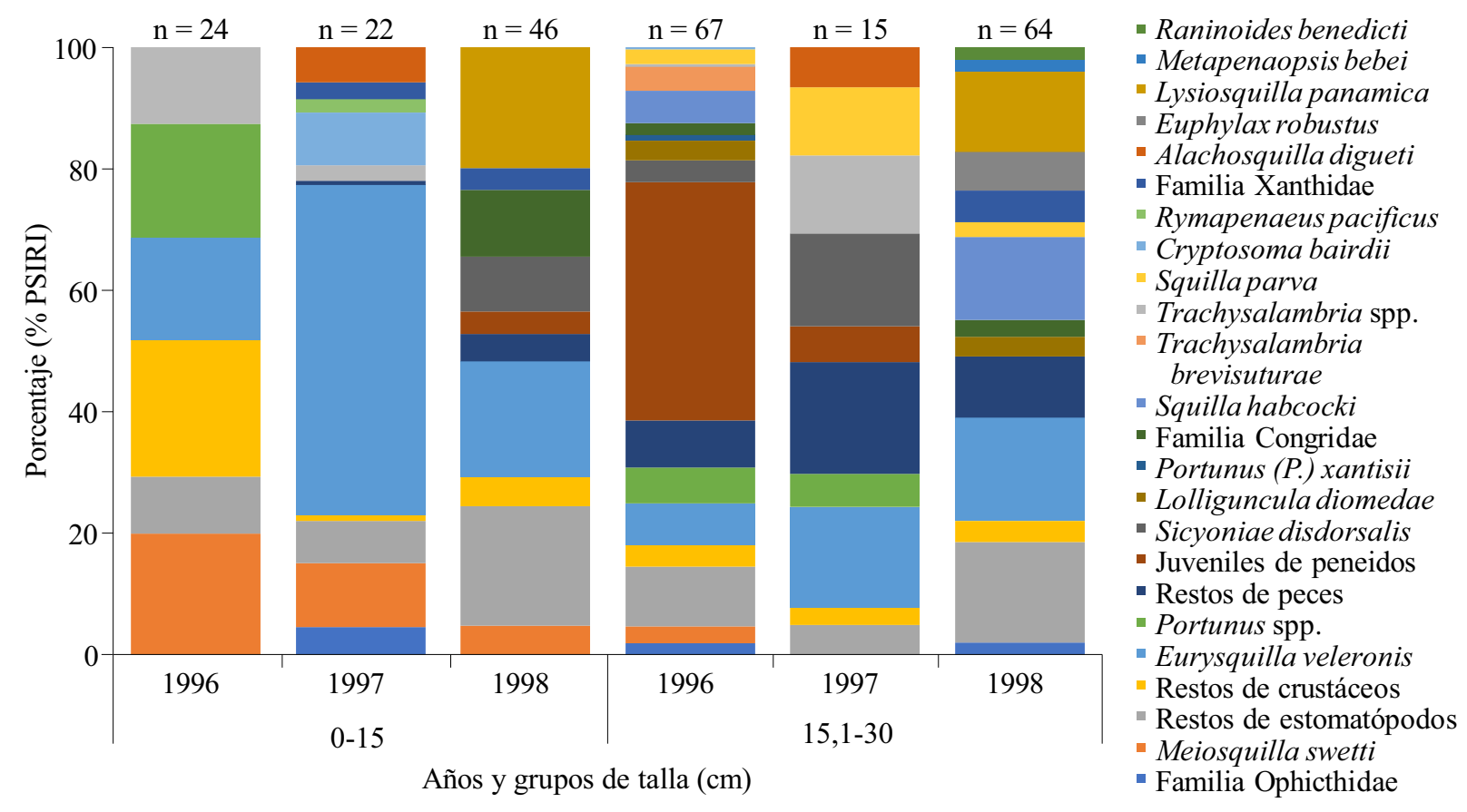

Figura 8. Porcentaje del índice de importancia presa específica (\%PSIRI), con respecto a los grupos de talla (cm LT) de Syacium latifrons durante los tres años de estudio.

Figure 8. Percentage of prey-specific index of relative importance (\%PSIRI) for food items of Syacium latifrons by length class (cm TL) during three years of study.

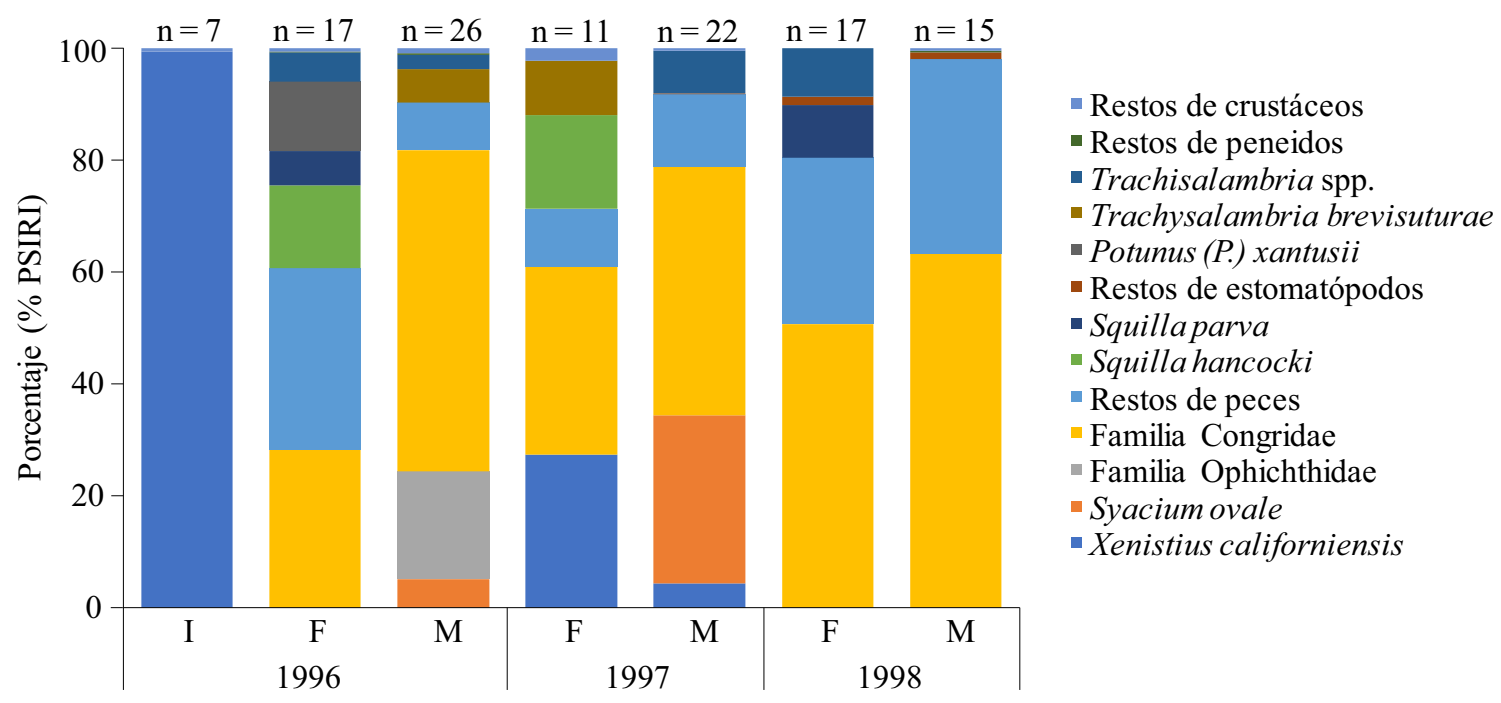

Años, hembras y machos

Figura 9. Porcentaje del índice de importancia presa específica (\%PSIRI), con respecto a hembras, machos e inmaduros de Cyclopsetta querna durante los tres años de estudio.

Figure 9. Percentage of prey-specific index of relative importance (\%PSIRI) for food items of Cyclopsetta querna by female, male, and immature class (cm TL) during three years of study. 


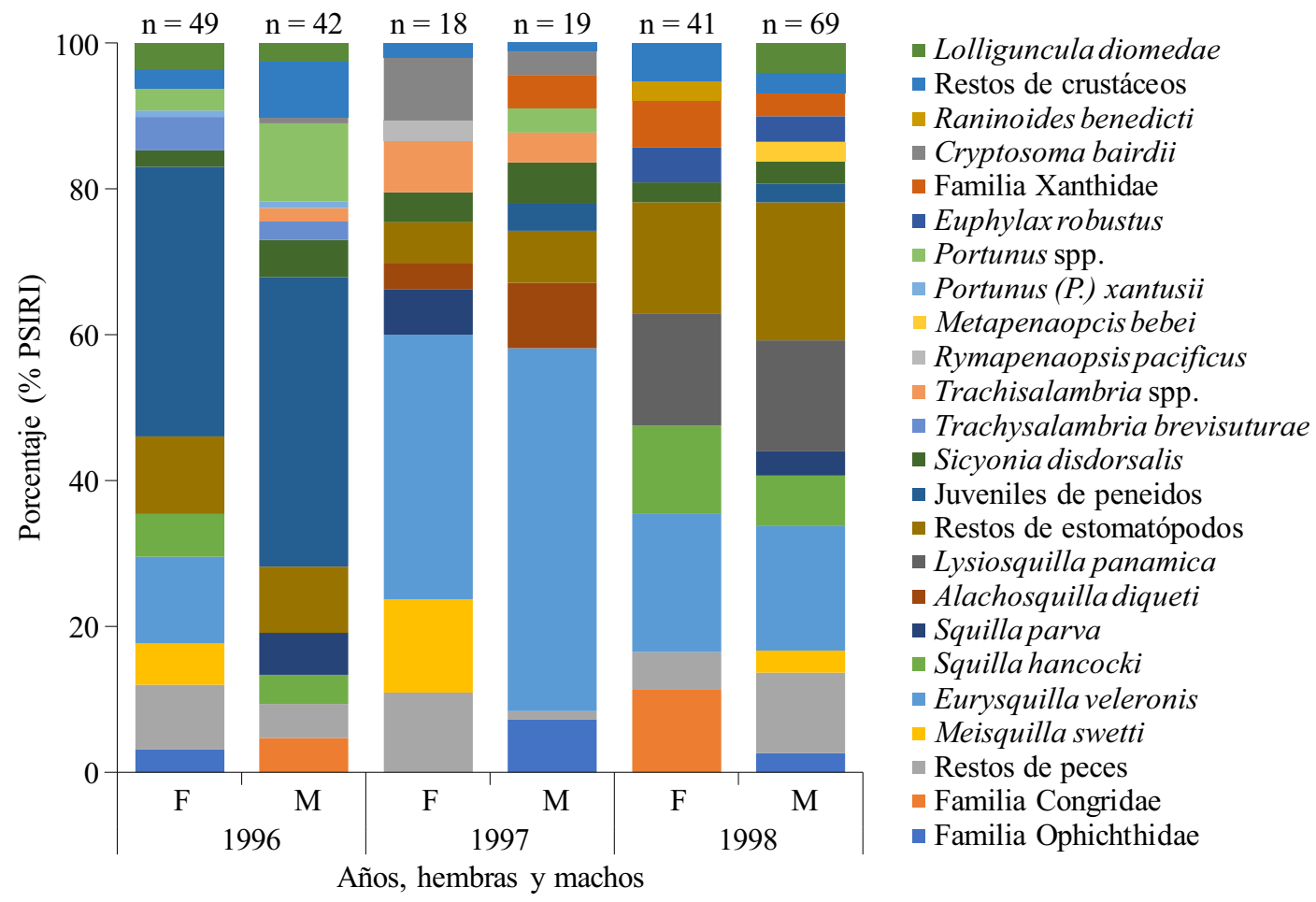

Figura 10. Porcentaje del índice de importancia presa específica (\%PSIRI), con respecto a hembras y machos de Syacium latifrons durante los tres años de estudio.

Figure 10. Percentage of prey-specific index of relative importance (\%PSIRI) for food items of Syacium latifrons by female, male, and immature class (cm TL) during three years of study.

Para 1996, S. latrifons se alimentó en primer término de los restos de estomatópodos $(30,01 \%)$, seguido de los restos de crustáceos $(25,01 \%)$, ambos considerados como alimento secundario. En 1997, E. veleronis es considerada como alimento preferencial $(64,54 \%)$ y los restos de estomatópodos como alimento frecuente $(16,03 \%)$. Para 1998 , lo restos de estomatópodos son considerados como alimento preferencial $(70,87 \%)$ y los restos de peces como alimento frecuente $(11,50 \%)$.

\section{Superposición de dietas}

Por último, el índice de traslape de dietas (Morisita) nos indica que no hay componentes alimentarios en común en la dieta de C. querna y $S$. latifrons, ya que los valores obtenidos se consideran muy bajos $(<0,5)$ en los tres años del estudio. Los valores obtenidos del índice de Morisita fueron de 0,4 en peso y 0 en número para 1996, 0,2 en peso y 0,1 en número para 1997 y de 0,4 en peso y 0,1 en número para 1998 .

\section{DISCUSIÓN}

Los ejemplares de C. querna y S. latifrons se encontraron distribuidos en la plataforma continental de Jalisco y Colima, México entre los 20 y $60 \mathrm{~m}$ de profundidad, lo cual es coincide con lo reportado por Mariscal Romero et al. (1998), quienes encontraron a estas dos especies entre los peces demersales más abundantes de la región a profundidades no mayores de $60 \mathrm{~m}$, ubicándose en el lugar séptimo y tercero de sus valores de 

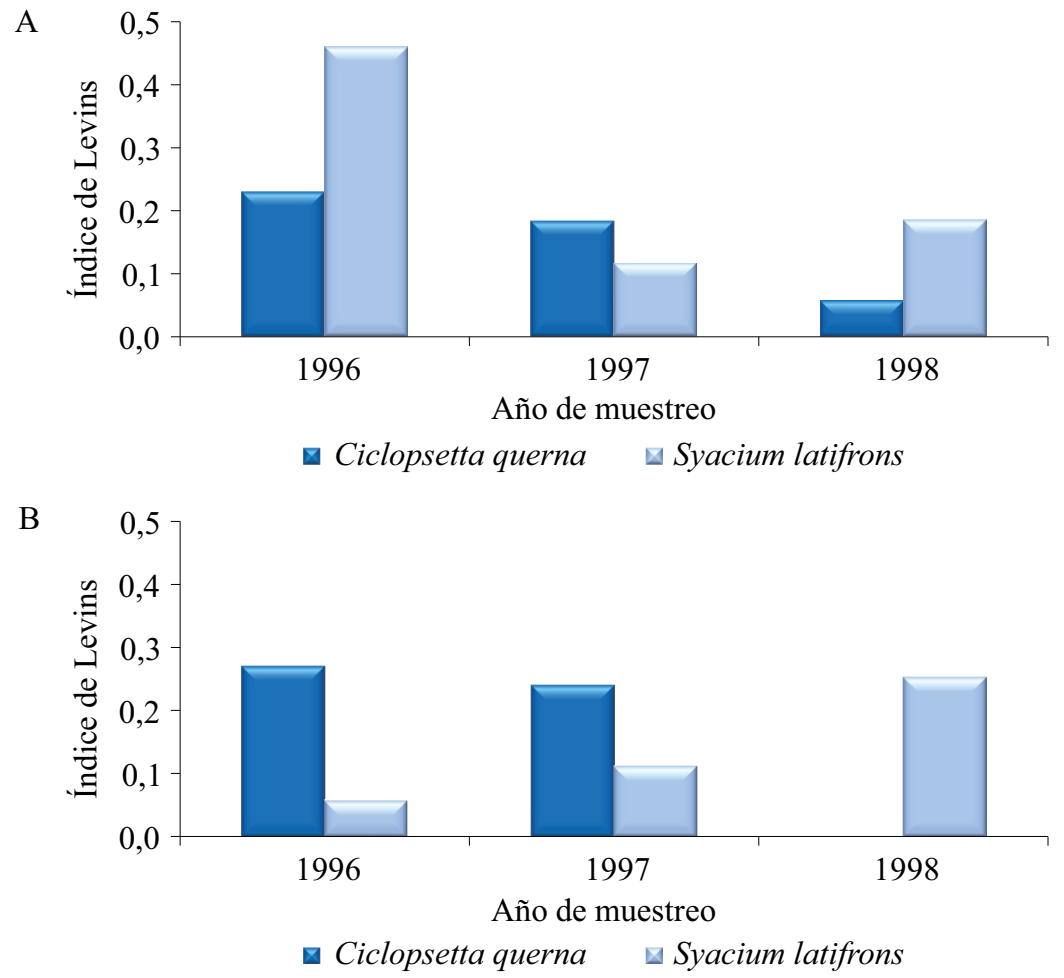

Figura 11. Diversidad anual (nicho trófico) en peso (A) y número de presas (B) de Ciclopsetta querna y Syacium latifrons. Figure 11. Annual diversity (trophic niche) in weight (A) and number (B) of prey of Ciclopsetta querna and Syacium latifrons.

abundancia, respectivamente. Rodríguez Ibarra (2006) considera a los lenguados como uno de los grupos más representativos de la ictiofauna demersal de los fondos blandos, ya que aportaron hasta el $44 \%$ de la captura total, presentando la mayor densidad a $60 \mathrm{~m}$ de profundidad. Por su parte, Flores Ortega et al. (2013) registraron para el Pacífico tropical mexicano a cinco especies de lenguados distribuidas en profundidades de $20 \mathrm{y}$ $60 \mathrm{~m}$, con la mayor abundancia y biomasa a los 20 m. Esta situación está determinada principalmente por la capacidad que tienen los lenguados para enterrarse en el sedimento, además de la abundancia de alimento y la necesidad de protegerse de los depredadores (Gibson 1994; Stoner y Abookire 2002).

En los contenidos estomacales de C. querna se identificaron 12 componentes alimentarios, mientras que para $S$. latifrons se identificaron 24 com- ponentes. Al respecto, Aarnio et al. (1996) identificaron en juveniles de lenguados 30 especies diferentes de presas, y Norbis y Galli (2004) identificaron en Paralichthys orbignyanus cinco ítems alimentarios. Por su parte, Amezcua y Portillo (2010) identificaron para $C$. panamensis un total de 12 grupos de presas. Amezcua et al. (2011) registraron para C. querna 14 presas diferentes, y Flores Ortega et al. (2013) identificaron 14 entidades alimentarias para esta misma especie y 30 categorías de presas para $S$. ovale. Los resultados obtenidos en este estudio son acordes con lo reportado por los autores arriba mencionados, destacándose la similitud en el número de presas identificado para C. querna.

Las presas principales de C. querna fueron los peces, y dentro de estos, los pertenecientes a las familias Congridae y Ophichthidae, y en segundo término los crustáceos. Amezcua et al. (2011) 
Tabla 3. Índice alimentario (IA) de Cyclopsetta querna y Syacium latifrons por año de estudio.

Table 3. Food index (IA) of Cyclopsetta querna and Syacium latifrons by year of study.

Cyclopseta querna

\begin{tabular}{lrrr}
\cline { 2 - 3 } & 1996 & 1997 & 1998 \\
\cline { 2 - 4 } Especies presa & IA & IA & IA \\
\hline Peces & & & 0 \\
Xenistius californiensis & 2,27 & 1,52 & 0 \\
Syacium ovale & 1,37 & 8,41 & 0 \\
Familia Ophichthidae & 6,19 & 0 & 12.91 \\
Familia Congridae & 43,47 & 33,06 & 86,13 \\
Restos de peces & 42,35 & & 0 \\
& & 0,21 & 0,09 \\
Estomatópodos & & 0 & 0,79 \\
Squilla hancocki & 0,61 & 0,03 &
\end{tabular}

Portunidos

Potunus (P) xantusii

0,16

0

Peneidos

Trachysalambria brevisuturae

0,23

0,04

0

Trachisalambria spp.

0,06

0,68

0,02

Restos de peneidos

0,37

0

0,01

Restos de crustáceos

2,85

1,62

0,05

Syacium latifrons

Peces

Familia Ophichthidae

0,31

0,46

0,39

0,01

Familia Congridae

17,4

0

0,44

Restos de peces

10,21

11,5

Estomatópodos

Meisquilla swetti

1,12

2,13

0,03

Eurysquilla veleronis

4,04

64,54

4,86

Squilla hancocki

1,51

0.00

1,36

Squilla parva

0,36

0,53

0,04 
Tabla 3. Continuación.

Table 3. Continued.

\begin{tabular}{|c|c|c|c|}
\hline \multirow[b]{3}{*}{ Especies presa } & \multicolumn{2}{|c|}{ Syacium latifrons } & \multirow[b]{2}{*}{1998} \\
\hline & 1996 & 1997 & \\
\hline & IA & IA & IA \\
\hline Alachosquilla diqueti & 0 & 1,28 & 0 \\
\hline Lysiosquilla panamica & 0 & 0 & 1,97 \\
\hline Restos de estomatópodos & 30,01 & 16,03 & 70,87 \\
\hline \multicolumn{4}{|l|}{ Peneidos } \\
\hline Juveniles de peneidos & 13,56 & 0,07 & 0,01 \\
\hline Sicyonia disdorsalis & 1,65 & 0,92 & 0,1 \\
\hline Trachysalambria brevisuturae & 1,15 & 0 & 0 \\
\hline Trachisalambria spp. & 0,07 & 1,06 & 0 \\
\hline Rymapenaopsis pacificus & 0 & 0,04 & 0 \\
\hline Metapenaopcis bebei & 0 & 0 & 0,01 \\
\hline \multicolumn{4}{|l|}{ Otros crustáceos } \\
\hline Portunus (P.) xantusii & 0,09 & 0 & 0 \\
\hline Portunus spp. & 2,08 & 0,04 & 0 \\
\hline Euphylax robustus & 0 & 0 & 0,12 \\
\hline Familia Xanthidae & 0 & 0,14 & 0,29 \\
\hline Cryptosoma bairdii & 0 & 0,28 & 0 \\
\hline Raninoides benedicti & 0 & 0 & 0,01 \\
\hline Restos de crustáceos & 25,01 & 2,34 & 8,3 \\
\hline \multicolumn{4}{|l|}{ Moluscos } \\
\hline Lolliguncula diomedae & 1,18 & 0 & 0,08 \\
\hline
\end{tabular}

mencionan que la dieta de C. querna estuvo dominada por peces demersales como Symphurus sp., y que son la fuente principal en su alimentación. Por su parte, Flores Ortega et al. (2013) reportaron que Manolene assaedae, Cyclopsetta panamensis y C. querna son consumidoras de peces e incluyen también a los crustáceos en su dieta.

En contraste, para $S$. latifrons las presas principales fueron los estomatópodos (E. veleronis y $M$. swetti). Amezcua y Portillo (2010) mencionan que $C$. panamensis es un consumidor activo de crustáceos y peces, pero que muestra preferencia por los primeros, donde Squilla parva es la presa de mayor biomasa $(28,5 \%)$. Flores Ortega et al. (2013) reportan que Bothus leopardinus y $S$. ovale, son consumidores de micro-crustáceos y en menor proporción de peces, lo cual coincide con los resultados del presente estudio. 
Se observó una ligera variación en la alimentación con los cambios ontogénicos de C. querna, dado que los organismos pequeños se alimentan principalmente del grupo de restos de crustáceos y $X$. californiensis, mientras que los individuos medianos y grandes prefieren a los peces como alimento principal. En contraste, la dieta de los ejemplares pequeños del primer grupo de talla de $S$. latifrons estuvo dominada por los estomatópodos, y en el segundo grupo por los estomatópodos, juveniles de peneidos y restos de peces. Lo anterior concuerda con los resultados de otros autores (Matallanas 1982; Wootton 1998 y Teixeira et al. 2010), quienes mencionan variaciones ontogénicas en la dieta de cuatro especies de lenguados y que esto podría estar relacionado con los cambios en las variaciones de espacio y en tiempo de las presas. Por su parte, Troccoli (2011) menciona que Paralichthys patagonicus mostró siempre una preferencia por los peces pelágicos, sin embargo, los de tallas menores consumen principalmente moluscos y crustáceos. Autores como Gerking (1994), Patell y Potter (1998), López Carzola y Forte (2005) y Marques et al. (2009), mencionan que lo lenguados de tallas menores poseen habilidad para capturar presas pequeñas y con menor movilidad, que y capturan presas más grandes y móviles a medida que alcanzan mayor tamaño, comportamiento que puede ser atribuido también al aumento en tamaño de la boca del depredador (Stoner y Livingston 1984).

Con respecto a diferencias en la alimentación atribuibles al sexo, no se observaron diferencias entre la alimentación de hembras y machos de $C$. querna, dado que el grupo de presas principales fue el de peces en ambos sexos. El mismo patrón se observó en $S$. latifrons, ya que tanto las hembras como los machos consumieron principalmente estomatópodos y peneidos, con lo que se puede inferir que estos organismos se alimentan de presas que se distribuyen principalmente en el fondo. Lo anterior es similar a lo reportado por Amezcua et al. (2011) para C. querna en el Golfo de California, en donde tanto juveniles como adultos, machos y hembras se alimentan principalmente de peces. Por su parte, Amezcua y Portillo (2010) reportaron que la dieta de C. panamensis en el Golfo de California estuvo dominada por estomatópodos y camarones, y que no mostró diferencias en lo que respecta al tamaño, sexo o la profundidad en donde se encuentren.

Con respecto a la diversidad del espectro alimentario (amplitud de nicho) el cálculo del índice de Levins presentó valores $<0,5$ para las dos especies y durante los tres años de estudio, por lo que se puede concluir que ambas especies presentan cierto grado de especialización. Esto concuerda con lo expuesto por Amezcua et al. (2011), quienes encontraron valores para este índice de 0,13 para C. querna, e indicaron que la dieta de esta especie se centra solo en unas pocas presas disponibles del sistema que habita. En contraste, Flores Ortega et al. (2013) describieron a C. querna como una especie generalista en el Pacífico central tropical, con valores de amplitud de nicho de 0,65 y 0,79 , a pesar de que solo identificaron 14 categorías alimentarias. Lo anterior podría estar relacionado con lo expuesto por De Raedemaecker et al. (2011), quienes mencionan que la alimentación de los lenguados se da principalmente por la disponibilidad de los recursos en las estaciones del año y de la capacidad de captura de presas de cada especie.

Finalmente, en lo que respecta a la superposición de la dieta de $C$. querna con la dieta de $S$. latifrons, los valores obtenidos fueron en todos los casos menores a 0,5 , lo que indica que no hay competencia por el alimento entre estas especies de lenguados. Por su parte, Flores Ortega et al. (2013), encontraron valores de superposición de dieta de bajos a moderados en cinco especies de lenguados. Sin embargo, otros autores como Burke (1995) y Cabral et al. (2002), mencionan que la superposición de dietas en lenguados no causa necesariamente competencia interespecifica, ya que frecuentemente la superposición es minimizada por las diferencias en tiempo y espacio. Darnaude et al. (2001), mencionan que la 
reducción de competencia por el alimento entre lenguados en una misma zona es lo que les permite coexistir. Lasiak y McLachlan (1987) y Vinagre et al. (2005), reconocen que los lenguados consumen el alimento más abundante de una manera generalista y oportunista.

\section{AGRADECIMIENTOS}

Este estudio no hubiera sido posible sin la ayuda de la tripulación del Barco de Investigación Pesquera BIP-V de la Universidad de Guadalajara. Apreciamos también la ayuda de los estudiantes, investigadores y técnicos que participaron en los muestreos del Proyecto Demersales, del antiguo Centro de Ecología Costera. Alina B. Ortíz apoyo en la estructuración de los mapas y las figuras. Este estudio se realizó con el apoyo financiero del CUCSUR de la Universidad de Guadalajara.

\section{REFERENCIAS}

AARnio K, Bonsdorff E, Rosenback N. 1996. Food and feeding habits of juvenile flounder Platichthys flesus and turbot Scophthalmus maximus in the Aland Archipelago, Northern Baltic Sea. J Sea Res. 36 (3/4): 311-320.

Aceves-Medina G, Saldierna-Martínez JR, GONZÁLEZ EA. 2003. Distribution and abundance of Syacium ovale larvae (Pleuronectiformes: Paralichthyidae) in the Gulf of California. Rev Biol Trop. 51 (2): 561-570.

Allen GR, Robertson DR. 1994. Fishes of the tropical eastern Pacific. Honolulu: University of Hawaii Press. 332 p.

Alonso MK, Crespob EA, García NA, Pedraza SN, MariotTiC PA, MoraC NJ. 2002. Fishery and ontogenetic driven changes in the diet of the spiny dogfish. Squalus acanthias, in
Patagonian waters, Argentina. Environ Biol Fishes. 63:193-202.

Amezcua F, Portillo A. 2010. Hábitos alimenticios del lenguado panámico Cyclopsetta panamensis (Paralichthyidae) en el sureste del Golfo de California. Rev Biol Mar Oceanogr. 45 (2): 335-340.

Amezcua F, Portillo A, Amezcua Linares F. 2011. Feeding habits of the toothed flounder Cyclopsetta querna (Paralichthyidae) of the southeast Gulf of California. J Mar Biol Assoc UK. 91: 1225-1230.

Anuario Estadístico de Acuacultura y Pesca. 2018. Comisión Nacional de Acuacultura y Pesca (CONAPESCA). Mazatlán, Sinaloa, México. 293 p.

Bocanegra-Castillo N, Abitia Cardénas la, GÁlvan Magaña F. 2000. Espectro alimentario de la berrugata californiana Menticirrhus undulatus de Laguna Ojo de Liebre, baja California Sur, México. Cienc Mar. 26 (4): 659675.

Brown SC, Bizzarro JJ, Cailliet GM, Ebert DA. 2012. Breaking with tradition: redefining measures for diet description with a case study of the Aleutian skate Bathyraja aleuca (Gilbert 1896). Environ Biol Fishes. 95 (1): 320.

BRUSCA RC. 1980. Common intertidal invertebrates of the Gulf of California. 2da ed. Tucson: University of Arizona Press. 153 p.

BURKE JS. 1995. Role of feeding and prey distribution of summer and southern flounders in selection of estuarine nursery habitats. J Fish Biol. 47: 355-366.

Cabral HN, Lopes M, Loeper R. 2002. Trophic niche overlap between flatfishes in a nursery area on the Portuguese coast. Sci Mar. 66 (3): 293-300.

Colwell RK. 2019. EstimaS: Statistical estimation of species richness and shared species from simples. Version S9.1.0. [consultado 10 febrero]. http://viceroy.eeb.uconn.edu/ estimates/. 
DANIEL WW. 1997. Bioestadística. Base para el análisis de las ciencias de la salud. 4ta ed. Ciudad de México: Limusa. 878 p.

Darnaude AM, Harmelin Vivien ML, Salen PICARD C. 2001. Food portioning among flatfish (Pisces: Pleuronectiforms) juveniles in a Mediterranean coastal shallow area. J Mar Biol Assoc UK. 81: 119-127.

De Raedemaecker F, O'Connor I, Brophy D, BLACK A. 2011. Macrobenthic prey availability and the potential for food competition between 0-year group Pleuronectes platessa and Limanda limanda. J Fish Biol. 79: 19181939.

Figueiredo M, Morato T, Barreiros JP, AlfonSo P, SAntos RS. 2005. Feeding ecology of the White seabream, Diplodussargus, and the ballan wrasse, Labrusbergylta, in the Azores. Fish Res. 75: 107-119.

Flores Ortega JR, Godínez Domínguez E, GonzÁlez SAnsón G, Rojo VÁzquez JA, López Prado AC, Morales Jauregui MY. 2013. Abundancia, distribución, hábitos alimentarios e interacciones tróficas de cinco especies de lenguados (Pleuronectiformes) en el Pacífico central mexicano. Lat Am J Aquat Res. 41 (3): 423-439.

Fischer W, Krupp F, Schneider W, Sommer C, Carpenter KE, Niem VH. 1995. Guía FAO para la identificación de especies para los fines de la pesca, Pacífico Centro-Oriental. Vertebrados. Parte I. Vol. II. Roma: FAO. p. 647-1200.

GERKING SD. 1994. Feeding ecology of fish. First edition. Nueva York: Academic Press. 416 p.

GIBSON RN. 1994. Impact of habitat quality and quantity on the recruitment of juvenile flatfishes. J Sea Res. 32: 191-206.

HENDRICKX ME. 1995a. Camarones. En: FisCHER W, Krupp F, Schneider W, Sommer C, CarPENTER KE, NiEM VH, editores. Guía FAO para la identificación de especies para los fines de la pesca. Pacífico Centro-Oriental. Vol. I. Roma: FAO. p. 417-537.
HeNDRICKX ME. 1995b. Cangrejos. En: FisCHER W, Krupp F, Schneider W, Sommer C, CARPENTER KE, NiEM VH, editores. Guía FAO para la identificación de especies para los fines de la pesca. Pacífico Centro-Oriental. Vol. I. Roma: FAO. p. 565-636.

Hendrickx ME, Salgado-Barragán J. 1991. Los estomatópodos (Crustacea: Hoplocarida) del Pacífico mexicano. Inst Cienc del Mar y Limnol, Univ Nal Autón México, Publ Esp. 10: 1-200.

HesPenheIDEN HA. 1975. Ecología de los productores primarios en la Laguna de Celestúm, México. Patrones de variación espacial y temporal [tesis doctoral]. Universidad de Barcelona. $233 \mathrm{p}$.

HoRn HS. 1966. Measurement of overlap in comparative ecological studies. Amer Naturalist. 100: 419-424.

Khidir KT, Chapleau F, Renaud CB. 2005. Phylogenetic relationships of Cyclopsetta and Syacium (Pleuronectiformes: Paralichthyidae). Cybium. 29 (4): 321-326.

Kouamelan PE, Teugels GG, Gourene G, Van Der Audenaerde DFET, Ollevier F. 2000. Feeding habits of Mormyrops anguilloides (Mormyridae) in lacustrine and riverine habitats of a West African basin. Cybium. 24 (1): 67-79.

KREBS CJ. 1989. Ecological methodology. Nueva York: Harper and Row. 550 p.

Lagler KF, Bardach JE, Miller RR, May PAssino DR. 1984. Ictiología. 1ra ed. Ciudad de México: AGT Editor. 489 p.

Lasiak T, McLachlan A. 1987. Opportunistic utilization of mysid shoals by surf-zone teleosted. Mar Ecol Prog Ser. 37: 1-7.

LAUZANNE L. 1975. Régime alimentaire d'Hidrocyon forskali (Pisces: Characidae) dans le lac Tchad et ses tributaires. Cahiers ORSTOM, Série Hydrobiologie. 9 (2): 105-121.

LEVINS R. 1968. Evolution in changing environments. Some theoretical explorations. Princeton: Princeton University Press. 123 p. 
López Carzola A, Forte S. 2005. Food and feeding habits of flounder Paralicthys orbignyanus (Jenyns, 1842) in Bahia Blanca estuary, Argentina. Hydrobiología. 549: 25257.

López Martínez J, Herrera Valdivia E, Rodríguez Romero J, Hernández VÁzquez S. 2010. Peces de la fauna de acompañamiento en la pesca industrial de camarón en el Golfo de California, México. Int J Trop Biol. 58 (3): 925-942.

Ludwing JA, REYNOLDS JF. 1988. Statistical ecology: a primer on methods and computing. Nueva York: Wiley-Interscience Publication. $337 \mathrm{p}$.

MaguRRAN AE. 2004. Ecological diversity and its measurement. Nueva Jersey: Princeton University Press. 179 p.

Matallanas J. 1982. Estudio del régimen alimentario de Lampanyctus crodilus (Risso, 1810) (Pisces, Myctophidae) en las costas catalanas (Med. Occid.). Téthys. 10: 254-260.

mariscal Romero J, Aguilar Palomino B, LuCANO RAMíREZ G, RAYMUNDO HuIZAR AR, RODRÍGUEZ IBARRA LE, RUÍZ RAMírez S, GonzÁlez SAnsón G. 1998. Asociaciones de peces demersales de la plataforma continental de Colima y Jalisco, México (primavera, 1995). Cienc Mar. 24 (1): 35-54.

Marques JF, Teixeira CM, Pinheiro A, Peschke K, CABRAL HN. 2009. A multivariate approach to the feeding ecology of the channel flounder Syacium micrurum (Pisces: Pleuronectiformes), in Cape Verde. Eastern Atlantic. Cienc Mar. 35 (1): 15-27.

MorisitA M. 1959. Measuring interspecific association and similarity between communities. Memoirs of the Faculty of Science. Kyushu University Series E (Biology). 3: 65-80.

MuraKami T, AmaoKa K. 1992. Review of the genus Syacium (Paralichthydae) with the description of a new species from Ecuador and Colombia. Bull Fac Fish Hokkaido Univ. 43 (2): 61-95.
NELSON JS. 2006. Fishes of the world. Fourth edition. Hoboken, Nueva Jersey: John Wiley \& Sons. $601 \mathrm{p}$.

NiKOLSKY G. 1963. The ecology of fishes. Londres: Academic Press. 352 p.

Norbis W, Galli O. 2004. Hábitos de alimentación del lenguado Paralichthys orbignyanus (Valenciennes, 1842) en una laguna costera somera del Atlántico Sur: Rocha, Uruguay. Cienc Mar. 30 (4): 619-626.

Patell ME, Potter IC. 1998. Distributions, size compositions and diets of two abundant benthic ambush-feeding teleosts in coastal waters of south-western Australia. J Mar Biol Assoc UK. 78: 587-608.

Pérez Farfante I, Kensley B. 1997. Penaeoid and Sergestotoid shrimps and prawns of the world. Keys and diagnoses for the families and genera. Paris: Memoires du Museum National d'Histoire Naturelle. 175: 1-13.

Pinkas L, Oliphant MS, IVERson LK. 1971. Food habitats of albacore, bluefin tuna, and bonito in California waters. Calif Fish Game, Fish Bull. 152: 1-105.

RATHBUN MJ. 1930. The cancroid crabs of America of the families Eurylidae, Portunidae, Atelecyclidae, Cangridae and Xanthidae. Bulletin of the United States. National Museum, (152): 1-229.

Raymundo Huizar AR, SAucedo Lozano M. 2008. Hábitos alimentarios del pez triglido Prionotus ruscarius (Gilbert \& Starks, 1904) durante 1996, en las costas de Jalisco y Colima, México. Rev Biol Mar Oceanogr. 43 (1): 7-15.

Rodiles-Hernández R, GonzÁlez DíAz AA. Chan Sala C. 2005. Lista de peces continentales de Chiapas, México. Hidrobiológica. 15 (2): 245-253.

RODRÍGUeZ IbARRA LE. 2006. Distribución y abundancia de los lenguados en la plataforma continental de Jalisco y Colima, México (1995-1996). En: JiMÉNEZ QUIROZ MDC, ESPINO BARR E, editores. Los recursos pesqueros y 
acuícolas de Jalisco, Colima y Michoacán. Guadalajara: Instituto Nacional de la Pesca, SAGARPA. p. 196-208.

Rodríguez Romero J, Palacios Salgado DS, López Martínez J, Hernández VÁzQuez S, Ponce Díaz G. 2008. Composición taxonómica y relaciones zoogeográficas de los peces demersales de la costa occidental de Baja California Sur, México. Rev Biol Trop. 56 (4): 1765-1783.

Rojas MPA, Zapata PLA. 2006. Peces demersales del parque Nacional Natural Gorgona y su área de influencia, Pacífico colombiano. Biota Colombiana. 7 (2): 211-244.

Rosecchi E, Novaze Y. 1987. Comparaison de cinq índices alimentaires utilisés dans y' analyse des contenus stomacaux. Rev Trav Inst Pêches Marit. 49 (3-4): 11-123.

Stoner AW, Abookire AA. 2002. Sediment preferences and size-specific distribution of young of the year Pacific halibut in an Alaska nursery. J Fish Biol. 61: 540-559.

Stoner AW, Livingston RJ. 1984. Ontogenetic patterns in diet and feeding morphology in sympatric sparid fishes from seagrass meadows. Copeia. 174-187 p.
Sturges H. 1926. The choice of a class-interval. J. Amer. Statist Assoc. 21: 65-66.

Teixeira CM, Batista MI, CABral HN. 2010. Diet, growth and reproduction of four flatfishes on the Portuguese coast. Sci Mar. 74 (2): 223-233.

Tobón-López A, Rubio EA, Giraldo A. 2008. Composición y análisis taxonómico de la ictiofauna del Golfo de Tribugá, Pacífico norte Colombia. Lat Am J Aquat Res. 36 (1): 93-104.

Troccoli GH. 2011. Ecología trófica del lenguado Paralichthys patagonicus (Jordan, 1889) en el ecosistema costero argentino-uruguayo [tesis de licenciatura]. Mar del Plata: Facultad de Ciencias Exactas y Naturales, Universidad Nacional del Mar de Plata. 76 p.

Vinagre C, França S, Costa MJ, Cabral HN. 2005. Niche overlap between juvenile flatfishes, Platichthys flesus and Solea solea, in a southern European estuary and adjacent coastal waters. J Appl Ichthyol. 21: 114-120.

WootTOn RJ. 1990. Ecology of teleost fish. Londres: Chapman \& Hall. 392 p.

Wootton RJ. 1998. Feeding. En: Woоtтon RJ, editor. Ecology of teleost fishes. Dordrecht: Kluwer Academic Publishers. p. 27-62. 